\title{
GRA method based on spherical linguistic fuzzy Choquet integral environment and its application in multi-attribute decision-making problems
}

\author{
Shahzaib Ashraf ${ }^{1}\left[\right.$ (D) Saleem Abdullah ${ }^{1} \cdot$ Tahir Mahmood $^{2}$
}

Received: 14 July 2018 / Accepted: 30 September 2018 / Published online: 16 October 2018

(c) The Author(s) 2018

\begin{abstract}
The key objective of the proposed work in this paper is to introduce a new version of picture linguistic fuzzy set, so-called spherical linguistic fuzzy sets. The novel concept of spherical linguistic fuzzy set consists of linguistic term, positive, neutral and negative membership degrees which satisfies the conditions that the square sum of its membership degrees is less than or equal to 1 . In this paper, we investigate the basic operations of spherical linguistic fuzzy sets and discuss some related results. We extend operational laws of aggregation operators and propose spherical linguistic fuzzy Choquet integral weighted averaging (SLFCIWA) operator based on spherical fuzzy numbers. Further, the proposed SLFCIWA operator of spherical fuzzy number is applied to multi-attribute group decision-making problems. Also, we propose the GRA method to aggregate the spherical fuzzy information. To implement the proposed models, we provide some numerical applications of group decision-making problems. Also compared with the previous model, we conclude that the proposed technique is more effective and reliable.
\end{abstract}

Keywords Choquet integral · Spherical linguistic fuzzy Choquet integral weighted averaging (SLFCIWA) operator · GRA method

Mathematics Subject Classification 03E72

\section{Introduction}

Fuzzy set theory developed by Zadeh [37] plays an important role in decision making under uncertain environment. Various direct/indirect extensions of fuzzy set have been made and successfully applied in most of the problems of

Shahzaib Ashraf

shahzaibashraf@awkum.edu.pk

Saleem Abdullah

saleemabdullah@awkum.edu.pk

Tahir Mahmood

tahirbakhat@iiu.edu.pk

1 Department of Mathematics, Abdul Wali Khan University, Mardan, Pakistan

2 Department of Mathematics and Statistics, International Islamic University, Islamabad, Pakistan real-world situation. An important generalization of fuzzy set theory is the theory of intuitionistic fuzzy set (IFS), introduced by Attanssov [6], that includes a membership and a non-membership degree. In 2010, Zhao [39] propsed generalized aggregation operators for IFSs. Later on, in 1994 Attanssov [5] introduced interval-valued intuitionistic fuzzy set (IvIFS) by extending the membership degree and nonmembership degree to interval number. In 2013, Yager strengthened the concept of IFS by proposing Pythagorean fuzzy sets (PyFS) [35] which somehow enlarge the space of positive membership and negative membership by introducing some new condition that $0 \leq P^{2}(x)+N^{2}(x) \leq 1$. In 2014, Yager [36] presented an example to state the condition that a decision maker gives his support for membership of an alternative $\left(\frac{\sqrt{ } 3}{2}\right)$ and his against membership is $\left(\frac{1}{2}\right)$. This fulfils the condition that their sum is bigger than 1 and is not presented for IFS, but it is presented for PyFS since $\left(\frac{\sqrt{ } 3}{2}\right)^{2}+\left(\frac{1}{2}\right)^{2} \leq 1$. Clearly, PyFS is more flexible than IFS 
to deal with the imprecision and ambiguity in the practical MADM problems. For more studies, we refer to [15, 24 26].

Such hitches were considered in Yager Pythagorean structure by Cuong [10], and he proposed picture fuzzy sets (PFS) of the form $(P(x), I(x), N(x))$ where the elements in triplet represent satisfaction, abstain and dissatisfaction degrees, respectively, under the condition that $0 \leq P(x)+I(x)+N(x) \leq 1$. This structure of Cuong is considerably closer to human nature than that of earlier concepts. Cuong also established the relations and compositions and found the distance between picture fuzzy numbers, also he gave the concept of picture soft sets. Cuong [11] in 2015 introduced the fuzzy logic operators for picture fuzzy sets. Singh [28] in 2015 introduced the idea related to correlation coefficients for picture fuzzy sets. Cuong [13] in 2016 established the classification of representable, $t$-norm operators for PFSs. Son [29] in 2016 introduced the concept about generalized picture distance measure and gave its applications. Wei [34] in 2016 introduced the cosine similarity measures for PFSs. Ashraf et al. [2] proposed different approaches to deal with multi-attribute group decision-making problems. For more study we refer $[3,4,8,9,17]$.

There were certain problems, which could not be expressed in terms of numerical values. Experts proposed certain linguistic terms that proved helpful in reducing the real situation problems. The great deal of qualitative information arises in real decision-making problem, which is simply transferred by linguistic terms, like as "very good", "good", "fair", "bad" and "very bad", . In some earlier applications, linguistic terms were described for triangular fuzzy numbers [1]. To handle fuzzy risk analysis, Pei and Shi [25] acknowledged linguistic weighted aggregation operator. A multi-criteria linguistic decision-making model is given by Rodriguez, in which experts give their judgment by eliciting linguistic expression. Based on the dependent operator, Liu [21] in 2013 derived the intuitionistic linguistic generalized dependent ordered weighted averaging operator and intuitionistic linguistic generalized dependent hybrid weighted aggregation operator. Wang et al. [31] in 2014 defined the score and accuracy index and comparison rules between two intuitionistic linguistic numbers. In 2015, Chen et al. [12] proposed a new notion of linguistic intuitionistic fuzzy number. Hence, keeping encouragement from the evidence that PFSs have the extreme dominant capability to model the approximated and inconclusive information in natural-world applications. For more study, we refer to [19, 22-24].

Due to the motivation and inspiration of the above conversation in this paper, we propose spherical linguistic fuzzy set (SLFS) which is generalization of Pythagorean linguistic fuzzy set and picture linguistic fuzzy set. Adding neutral membership degree in Pythagorean fuzzy set or taking square of membership degrees of picture fuzzy set leads us to spherical linguistic fuzzy set. In SLFS, membership degrees are gratifying the condition $0 \leq P^{2}(x)+I^{2}(x)+N^{2}(x) \leq 1$. Dealing with MAGDM, in this situation, when the decision maker provides the positive, negative and neutral membership degrees of a particular attribute in such a way that their sum is greater than one, it is better to use spherical linguistic fuzzy information. Sometimes, the attribute values take the form of spherical linguistic fuzzy information and the information about attribute weights is completely unknown because of time stress, lack of data or knowledge, and the expert's limited knowledge about the problem domain. The classic GRA method [14], intuitionistic fuzzy GRA method $[32,33]$ and Pythagorean fuzzy GRA method will fail in dealing with the above spherical linguistic fuzzy MADM problems with completely unknown weight information. The interesting and important research in this article is to derive the attribute weights from both the given spherical linguistic fuzzy information and completely unknown attribute weight information based on the traditional GRA method. Therefore, it is essential to concentrate on this problem. The purpose of this paper is to develop the concept of GRA methodology for solving MADM problems under spherical linguistic fuzzy environment, in which the data about attribute weights are incompletely known, and the attribute values take the form of spherical linguistic fuzzy numbers. The remainder of the paper is structured as follows:

In "Preliminaries" section, some revision of the definitions and results of Choquet integral, Pythagorean linguistic fuzzy sets and Picture linguistic fuzzy sets is given. In "GRA method with incomplete weight information for spherical linguistic fuzzy setting" section, the concept of spherical linguistic fuzzy sets is proposed. The GRA method for spherical linguistic fuzzy MAGDM problems with incomplete weight information is stated in "GRA method with incomplete weight information for spherical linguistic fuzzy setting" section. In "Descriptive example" section, we explain our planned algorithm and proved with help of an example. Conclusion is given in "Conclusion" section.

\section{Preliminaries}

The section provides the review of basic definition and notations of linguistic variable, Pythagorean fuzzy sets (PyFSs), Pythagorean linguistic fuzzy sets (PLFSs), picture fuzzy sets (PFS), picture linguistic fuzzy sets (PLFS) and their operations. Concept related to fuzzy measure and the Choquet integral and some further ideas which will be utilized in the later analysis are also be discussed.

Definition 1 [5] Let $L=\left\{\zeta_{p} \mid p=0,1, \ldots, l\right\}$ be the linguistic term set and the cardinality be odd where $\zeta_{p}$ is the possible value for a linguistic variable and $l$ is a positive integer. That is, a seven linguistic terms set $L$ could be assigned as: 
$L=\left(\zeta_{0}, \zeta_{1}, \zeta_{2}, \zeta_{3}, \zeta_{4}, \zeta_{5}, \zeta_{6}\right)=\{$ verypoor, poor, slightly poor, fair, slightlygood, good, verygood $\}$. Consistently, in such a cases, the following characteristics should be held in linguistic term set.

(1) The negation operator: neg $\left(L_{p}\right)=L_{q}$, where $q=l-1$;

(2) Be ordered: $\zeta_{p} \leq \zeta_{q} \Longleftrightarrow p \leq q$;

(3) Maximum operator: $\max \left(\zeta_{p}, \zeta_{q}\right)=\zeta_{p}$ if $\zeta_{p} \geq \zeta_{q}$;

(4) Minimum operator: $\min \left(\zeta_{p}, \zeta_{q}\right)=\zeta_{p}$ if $\zeta_{p} \leq \zeta_{q}$.

$\zeta_{[0, l]}=\left\{\zeta_{p} \mid \zeta_{0} \leq \zeta_{p} \leq \zeta_{l}\right\}$, all the above characteristics also get the elements; if $\zeta_{p} \in L$, then it is said as the actual term; otherwise, $\zeta_{p} \in L$, it is said as the virtual term. To maintain all the given information, Herrera et al. [16] assign the distinct linguistic title $L=\left(l_{0}, l_{1}, \ldots, l_{l-1}\right)$ is spread to a continuous linguistic title $L=\left\{l_{\theta} \mid \theta \in(0, R)\right\}$, where $R$ is the adequately large positive real number which satisfies the upper characteristics.

Definition 2 [5] The operational laws of linguistic variables $l_{p}, l_{q} \in L$ are defined as:

(1) $\vartheta \otimes l_{p}=l_{\vartheta \cdot p}$

(2) $l_{p} \oplus l_{q}=l_{p+q}$

(3) $l_{p} / l_{q}=l_{p / q}$

(4) $\left(l_{p}\right)^{q}=l_{p^{q}}$

Definition 3 [35] A PyFS $\breve{E}_{\breve{u}}$ on the universe of discourse $\mathbb{R} \neq \phi$ is defined as:

$\breve{E}_{\breve{u}}=\left\{\left\langle P_{\breve{e}_{\breve{u}}}(r), I_{\breve{e}_{\breve{u}}}(r) \mid r \in \mathbb{R}\right\rangle\right\}$.

A PyFS in a set $\mathbb{R}$ is indicated by $P_{\breve{e}_{\breve{u}}}(r): \mathbb{R} \rightarrow[0,1]$ and $I_{\check{e}_{\breve{u}}}(r): \mathbb{R} \rightarrow[0,1]$ which are the positive and negative membership degrees of $\breve{E}_{\breve{u}}$ in $\mathbb{R}$, respectively. Furthermore $P_{\breve{e}_{\breve{u}}}(r)$ and $I_{\check{e}_{\breve{u}}}(r)$ satisfy $0 \leq P_{\breve{e}_{\breve{u}}}^{2}(r)+I_{\check{e}_{\breve{u}}}^{2}(r) \leq 1$ for all $r \in \mathbb{R}$.

Definition 4 [10] A PFS $\breve{E}_{\breve{u}}$ on the universe of discourse $\mathbb{R} \neq \phi$ is defined as:

$\breve{E}_{\breve{u}}=\left\{\left\langle P_{\breve{e}_{\breve{u}}}(r), I_{\breve{e}_{\breve{u}}}(r), N_{\check{e}_{\breve{u}}}(r) \mid r \in \mathbb{R}\right\rangle\right\}$.

A PFS in a set $\mathbb{R}$ is indicated by $P_{\breve{e}_{\breve{u}}}(r): \mathbb{R} \rightarrow[0,1]$, $I_{\check{e}_{\breve{u}}}(r): \mathbb{R} \rightarrow[0,1]$ and $N_{\breve{e}_{\breve{u}}}(r): \mathbb{R} \rightarrow[0,1]$ which are the positive, neutral and negative membership degrees of $\breve{E}_{\breve{u}}$ in $\mathbb{R}$, respectively. Furthermore $P_{\breve{e}_{\breve{u}}}(r), I_{\check{e}_{\breve{u}}}(r)$ and $N_{\check{e}_{\breve{u}}}(r)$ satisfy $0 \leq P_{\check{e}_{\breve{u}}}(r)+I_{\check{e}_{\breve{u}}}(r)+N_{\check{e}_{\breve{u}}}(r) \leq 1$ for all $r \in \mathbb{R}$.

Definition 5 [27] A PLFS $\breve{E}_{\breve{u}}$ on the universe of discourse $\mathbb{R} \neq \phi$ is defined as:

$\breve{E}_{\breve{u}}=\left\{\left\langle\zeta_{\check{e}_{\breve{u}}}(r), P_{\breve{e}_{\breve{u}}}(r), I_{\check{e}_{\breve{u}}}(r), N_{\check{e}_{\breve{u}}}(r) \mid r \in \mathbb{R}\right\rangle\right\}$.
A PLFS in a set $\mathbb{R}$ is indicated by $\zeta_{\check{e}_{\breve{u}}}(r) \in L$, $P_{\check{e}_{\breve{u}}}(r): \mathbb{R} \rightarrow[0,1], \quad I_{\check{e}_{\breve{u}}}(r): \mathbb{R} \rightarrow[0,1] \quad$ a n d $N_{\breve{e}_{\breve{u}}}(r): \mathbb{R} \rightarrow[0,1]$ which are the linguistic term, the positive, neutral and negative membership degrees of $\breve{E}_{\breve{u}}$ in $\mathbb{R}$, respectively. Furthermore $P_{\check{e}_{\breve{u}}}(r), I_{\check{e}_{\breve{u}}}(r)$ and $N_{\check{e}_{\breve{u}}}(r)$ satisfy $0 \leq P_{\breve{e}_{\breve{u}}}(r)+I_{\breve{e}_{\breve{u}}}(r)+N_{\check{e}_{\breve{u}}}(r) \leq 1$ for all $r \in \mathbb{R}$.

\section{Fuzzy measure and Choquet integral}

Definition 6 [7] Let the universe of discourse $\mathbb{R}=\left\{r_{1}, r_{2}, \ldots, r_{n}\right\}$ and $p(\mathbb{R})$ represent the power set of $\mathbb{R}$. A fuzzy measure $P_{\breve{e}_{\breve{u}}}$ on $\mathbb{R}$ is a set function $P_{\breve{e}_{\breve{u}}}: p(\mathbb{R}) \rightarrow[0,1]$, which satisfy the following conditions:

(1) $P_{\breve{e}_{\breve{u}}}(\phi)=0, P_{\breve{e}_{\breve{u}}}(\mathbb{R})=1$.

(2) If $\quad \breve{E}_{\breve{u}_{1}}, \breve{E}_{\breve{u}_{2}} \in p(\mathbb{R}) \quad$ a n d $\quad \breve{E}_{\breve{u}_{1}} \subseteq \breve{E}_{\breve{u}_{2}}$, the n $P_{\breve{e}_{\breve{u}}}\left(\breve{E}_{\breve{u}_{1}}\right) \leq P_{\breve{e}_{\breve{u}}}\left(\breve{E}_{\breve{u}_{2}}\right)$.

Still, it is needed to sum the axiom of continuity; when $\mathbb{R}$ is infinite, it is sufficient to assume a finite universal set in definite practice. $P_{\breve{e}_{\breve{u}}}\left(\left\{r_{1}, r_{2}, \ldots, r_{n}\right\}\right)$ is the class of subjective importance of decision attribute set $\left\{r_{1}, r_{2}, \ldots, r_{n}\right\}$. Thus, with the separate weights of attributes, weights of any combination of attributes can also be defined. Naturally, we could say the following about any pair of criteria sets $\breve{E}_{\breve{u}_{1}}, \breve{E}_{\breve{u}_{2}} \in p(\mathbb{R}), \breve{E}_{\breve{u}_{1}} \cap \breve{E}_{\breve{u}_{2}}=\phi ; \breve{E}_{\breve{u}_{1}}$ and $\breve{E}_{\breve{u}_{2}}$ are considered to be without communication (or to be independent) if

$P_{\breve{e}_{\breve{u}}}\left(\breve{E}_{\breve{u}_{1}} \cup \breve{E}_{\breve{u}_{2}}\right)=P_{\breve{e}_{\breve{u}}}\left(\breve{E}_{\breve{u}_{1}}\right)+P_{\breve{e}_{\breve{u}}}\left(\breve{E}_{\breve{u}_{2}}\right)$

which is called an additive measure. $\breve{E}_{\breve{u}_{1}}$ and $\breve{E}_{\breve{u}_{2}}$ model a positive synergetic interaction between them (or are complementary) if

$P_{\breve{e}_{\breve{u}}}\left(\breve{E}_{\breve{u}_{1}} \cup \breve{E}_{\breve{u}_{2}}\right)>P_{\breve{e}_{\breve{u}}}\left(\breve{E}_{\breve{u}_{1}}\right)+P_{\breve{e}_{\breve{u}}}\left(\breve{E}_{\breve{u}_{2}}\right)$

which is called a super-additive measure. $\breve{E}_{\breve{u}_{1}}$ and $\breve{E}_{\breve{u}_{2}}$ exhibits a negative synergetic interaction between them (or are redundant or substitutive) if

$P_{\breve{e}_{\breve{u}}}\left(\breve{E}_{\breve{u}_{1}} \cup \breve{E}_{\breve{u}_{2}}\right)<P_{\breve{e}_{\breve{u}}}\left(\breve{E}_{\breve{u}_{1}}\right)+P_{\breve{e}_{\breve{u}}}\left(\breve{E}_{\breve{u}_{2}}\right)$

which is said to be a sub-additive measure. After all it is difficult to find the fuzzy measure according to Definition 6 and thus to confirm a fuzzy measure in MAGDM problems; Sugeno [30] admits the following $\lambda$-fuzzy measure:

$P_{\breve{e}_{\breve{u}}}\left(\breve{E}_{\breve{u}_{1}} \cup \breve{E}_{\breve{u}_{2}}\right)=P_{\breve{e}_{\breve{u}}}\left(\breve{E}_{\breve{u}_{1}}\right)+P_{\breve{e}_{\breve{u}}}\left(\breve{E}_{\breve{u}_{2}}\right)+\lambda P_{\breve{e}_{\breve{u}}}\left(\breve{E}_{\breve{u}_{1}}\right) P_{\breve{e}_{\breve{u}}}\left(\breve{E}_{\breve{u}_{2}}\right)$

$\lambda \in[-1, \infty), \breve{E}_{\breve{u}_{1}} \cap \breve{E}_{\breve{u}_{2}}=\phi$. The parameter $\lambda$ determines interaction between the attributes. In Eq. (2.7), if $\lambda=0, \lambda$ -fuzzy measure becomes simply an additive measure. And 
for negative and positive $\lambda$, the $\lambda$-fuzzy measure become to sub-additive and super-additive measures, respectively. Meantime, if all the elements in $\mathbb{R}$ are independent, and we have

$$
P_{\breve{e}_{\breve{u}}}\left(\breve{E}_{\breve{u}}\right)=\sum_{p=1}^{n} P_{\breve{e}_{\breve{u}}}\left(\left\{r_{p}\right\}\right)
$$

If $\mathbb{R}$ is a finite set, then $\cup_{p=1}^{n} r_{p}=\mathbb{R}$. The $\lambda$-fuzzy measure $P_{\check{e}_{\ddot{u}}}$ satisfies Eq. (2.9)

$P_{\breve{e}_{\breve{u}}}(\mathbb{R})=P_{\check{e}_{\grave{u}}}\left(\cup_{p=1}^{n} r_{i}\right)= \begin{cases}\frac{1}{\lambda}\left(\prod_{p=1}^{n}\left[1+\lambda P_{\check{e}_{\grave{u}}}\left(r_{p}\right)\right]-1\right) & \text { if } \lambda \neq 0 \\ \sum_{p=1}^{n} P_{\check{e}_{\grave{u}}}\left(r_{p}\right) & \text { if } \lambda=0\end{cases}$

where $r_{p} \cap r_{\breve{d}}=\phi$ for all $p, \breve{d}=1,2, \ldots, n$ and $p \neq \breve{d}$. It can be noted that $P_{\breve{e}_{\breve{u}}}\left(r_{p}\right)$ for a subset with a single element $r_{p}$ is said to be a fuzzy density and can be represented as $P_{\check{\tilde{u}}_{\breve{u}_{p}}}=P_{\check{e}_{\grave{u}}}\left(r_{p}\right)$. Specially for every subset $\breve{E}_{\breve{u}_{1}} \in p(\mathbb{R})$, we have

$P_{\check{e}_{\breve{u}}}\left(\breve{E}_{\breve{u}_{1}}\right)= \begin{cases}\frac{1}{\lambda}\left(\prod_{p=1}^{n}\left[1+\lambda P_{\check{e}_{\breve{u}}}\left(r_{p}\right)\right]-1\right) & \text { if } \lambda \neq 0 \\ \sum_{p=1}^{n} P_{\breve{e}_{\breve{u}}}\left(r_{p}\right) & \text { if } \lambda=0\end{cases}$

Based on Eq. (2.5), the value of $\lambda$ can be uniquely found from $P_{\breve{e}_{\breve{u}}}(\mathbb{R})=1$, which is equivalent to solving

$\lambda+1=\prod_{p=1}^{n}\left[1+\lambda P_{\check{e}_{\tilde{u}_{p}}}\right]$

It also noticed that $\lambda$ can be uniquely determined by $P_{\check{e}_{\breve{u}}}(\mathbb{R})=1$.

Definition 7 [30] Let $f$ and $P_{\breve{e}_{\breve{\mu}}}$ be a positive real-valued function and fuzzy measure on $\mathbb{R}$, respectively. With respect to $P_{\check{e}_{\breve{u}}}$, the discrete Choquet integral of $f$ is defined as

$C_{\mu}(f)=\sum_{p=1}^{n} f_{\rho(p)}\left[P_{\check{e}_{\breve{u}}}\left(A_{\rho(p)}\right)-P_{\check{e}_{\breve{u}}}\left(A_{\rho(p-1)}\right)\right]$

where $\rho(p)$ represents a permutation on $\mathbb{R}$, where $f_{\rho(1)} \geq f_{\rho(2)} \geq \cdots \geq f_{\rho(n)}, A_{\rho(n)}=\{1,2, \ldots, p\}, A_{\rho(0)}=\phi$.

\section{Spherical linguistic fuzzy set and its operations}

In this section, the concept of SFS, SLFS and their operational laws are developed.

Definition 8 A SFS $\breve{E}_{\breve{u}}$ on the universe of discourse $\mathbb{R} \neq \phi$ is defined as:

$\breve{E}_{\breve{u}}=\left\{\left\langle P_{\breve{e}_{\breve{u}}}(r), I_{\check{e}_{\breve{u}}}(r), N_{\breve{e}_{\breve{u}}}(r) \mid r \in \mathbb{R}\right\rangle\right\}$.

A PFS in a set $\mathbb{R}$ is indicated by $P_{\breve{e}_{\tilde{u}}}(r): \mathbb{R} \rightarrow[0,1]$, $I_{\check{e}_{\tilde{u}}}(r): \mathbb{R} \rightarrow[0,1]$ and $N_{\check{e}_{\tilde{u}}}(r): \mathbb{R} \rightarrow[0,1]$ which are the positive, neutral and negative membership degrees of $\breve{E}_{\breve{u}}$ in $\mathbb{R}$, respectively. Furthermore $P_{\check{e}_{\tilde{u}}}(r), I_{\check{e}_{\breve{u}}}(r)$ and $N_{\check{e}_{\tilde{u}}}(r)$ satisfy $0 \leq P_{\check{e}_{\breve{u}}}^{2}(r)+I_{\check{e}_{\breve{u}}}^{2}(r)+N_{\check{e}_{\tilde{u}}}^{2}(r) \leq 1$ for all $r \in \mathbb{R}$.

$$
\chi_{\breve{E}_{\breve{u}}}(r)=\sqrt{1-\left(P_{\check{e}_{\breve{u}}}^{2}(r)+I_{\check{e}_{\ddot{u}}}^{2}(r)+N_{\check{e}_{\breve{u}}}^{2}(r)\right)} \text { is said to be }
$$

refusal degree of $r$ in $\mathbb{R}$, for SFS $\left\{\left\langle P_{\check{e}_{\breve{u}}}(r), I_{\check{e}_{\breve{u}}}(r), N_{\check{e}_{\breve{u}}}(r) \mid r \in \mathbb{R}\right\rangle\right\}$, whose triple components $\left\langle P_{\check{e}_{\breve{u}}}, I_{\breve{e}_{\breve{u}}}, N_{\check{e}_{\breve{u}}}\right\rangle$ are said to SFN and each SFN can be denoted by $E=\left\langle P_{e}, I_{e}, N_{e}\right\rangle$, where $P_{e}, I_{e}$ and $N_{e} \in[0,1]$, with condition that $0 \leq P_{e}^{2}+I_{e}^{2}+N_{e}^{2} \leq 1$.

Spherical fuzzy sets have its own importance in a circumstance where opinion is not only constrained to yes or no, but there is some sort of abstinence or refusal. A good example of spherical fuzzy set could be decision making such as when four decision makers have four different categories of opinion about a candidate. Another example could be of voting where there are four types of voters who vote in favour or vote against or refuse to vote or abstain. Spherical fuzzy set is a direct generalization of fuzzy set, intuitionistic fuzzy set and picture fuzzy set.

A question arises that why we need spherical fuzzy set or what are the boundaries of PFSs that lead us to spherical fuzzy sets? The main downside of PFSs is the restriction on it, i.e., $0 \leq P_{\check{e}_{\breve{u}}}(r)+I_{\check{e}_{\breve{u}}}(r)+N_{\check{e}_{\breve{u}}}(r) \leq 1$ as this condition does not allow the decision makers to assign membership values of their own consent. The decision makers are somehow limitations in a specific domain. We consider an example $P_{\breve{e}_{\breve{u}}}=0.8, I_{\breve{e}_{\breve{u}}}=0.5$ and $N_{\check{e}_{\breve{u}}}=0.3$ which interrupts the condition that $0 \leq P_{\check{e}_{\breve{u}}}+I_{\check{e}_{\breve{u}}}+N_{\check{e}_{\breve{u}}} \leq 1$, but if we take the square of these values such as $P_{\check{e}_{\tilde{u}}}^{2}=0.64, I_{\check{e}_{\tilde{u}}}^{2}=0.25$ and $N_{\check{e}_{\breve{u}}}^{2}=0.09$ where the condition $0 \leq P_{\check{e}_{\breve{u}}}^{2}+I_{\check{e}_{\breve{u}}}^{2}+N_{\check{e}_{\breve{u}}}^{2} \leq 1$ is satisfied.

Note that if we put $I_{\check{e}_{\breve{u}}}=0$ in SPSs. Then, SPSs reduced to pythagorean fuzzy sets. Hence, spherical fuzzy sets are direct extension of pythagorean fuzzy set and also the extension of picture fuzzy sets, and also we seen the hierarchy structure (below) of the spherical fuzzy sets (Fig. 1). 


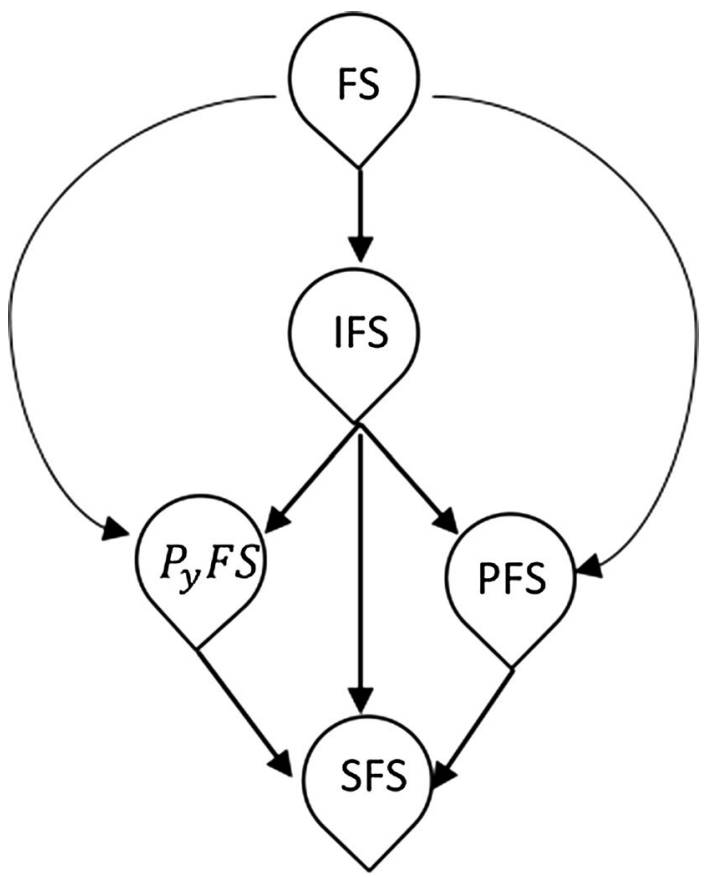

Fig. 1 Hierarchy structure of spherical fuzzt set

Definition 9 A SLFS $\breve{E}_{\breve{u}}$ on the universe of discourse $\mathbb{R} \neq \phi$ is defined as:

$\breve{E}_{\breve{u}}=\left\{\left\langle\zeta_{\breve{e}_{\breve{u}}}(r), P_{\breve{e}_{\breve{u}}}(r), I_{\breve{e}_{\breve{u}}}(r), N_{\breve{e}_{\breve{u}}}(r) \mid r \in \mathbb{R}\right\rangle\right\}$.

A SLFS in a set $\mathbb{R}$ is indicated by $\zeta_{\check{e}_{\bar{u}}}(r) \in L$, $P_{\breve{e}_{\tilde{u}}}(r): \mathbb{R} \rightarrow[0,1] \quad, \quad I_{\check{e}_{\tilde{u}}}(r): \mathbb{R} \rightarrow[0,1] \quad$ a n d $N_{\check{e}_{\tilde{u}}}(r): \mathbb{R} \rightarrow[0,1]$ which are the linguistic term, the positive, neutral and negative membership degrees of each $r \in \mathbb{R}$, respectively. Furthermore, $P_{\check{e}_{\grave{u}}}(r), I_{\check{e}_{\breve{u}}}(r)$ and $N_{\check{e}_{\grave{u}}}(r)$ satisfy $0 \leq P_{\grave{e}_{\tilde{u}}}^{2}(r)+I_{\check{e}_{\tilde{u}}}^{2}(r)+N_{\check{e}_{\tilde{u}}}^{2}(r) \leq 1$ for all $r \in \mathbb{R}$. $\chi_{\breve{E}_{\breve{u}}}(r)=\sqrt{1-\left(P_{\check{e}_{\grave{u}}}^{2}(r)+I_{\breve{e}_{\breve{u}}}^{2}(r)+N_{\check{e}_{\breve{u}}}^{2}(r)\right)}$ is said to be refusal degree of $r$ in $\mathbb{R}$, for SFS $\left\{\left\langle P_{\breve{e}_{\breve{u}}}(r), I_{\check{e}_{\breve{u}}}(r), N_{\check{e}_{\breve{u}}}(r) \mid r \in \mathbb{R}\right\rangle\right\}$, whose triple components

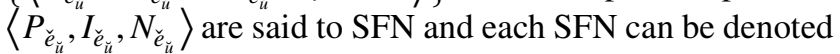
by $E=\left\langle P_{e}, I_{e}, N_{e}\right\rangle$, where $P_{e}, I_{e}$ and $N_{e} \in[0,1]$, with condition that $0 \leq P_{e}^{2}+I_{e}^{2}+N_{e}^{2} \leq 1$ (Fig. 2).

Definition 10 Let $\breve{E}_{\breve{u}_{1}}=\left\langle\zeta_{\check{e}_{\check{u}_{1}}}, P_{\check{e}_{\breve{u}_{1}}}, I_{\check{e}_{\breve{u}_{1}}}, N_{\check{e}_{\breve{u}_{1}}}\right\rangle$ and $\breve{E}_{\breve{u}_{2}}=\left\langle\zeta_{\check{e}_{\check{u}_{2}}}, P_{\check{e}_{\check{u}_{2}}}, I_{\check{e}_{\check{u}_{2}}}, N_{\check{e}_{\check{u}_{2}}}\right\rangle$ be two SLFNs defined on the universe of discourse $\mathbb{R} \neq \phi$, and some operations on SLFNs are defined as follows with $\tau \geq 0$.

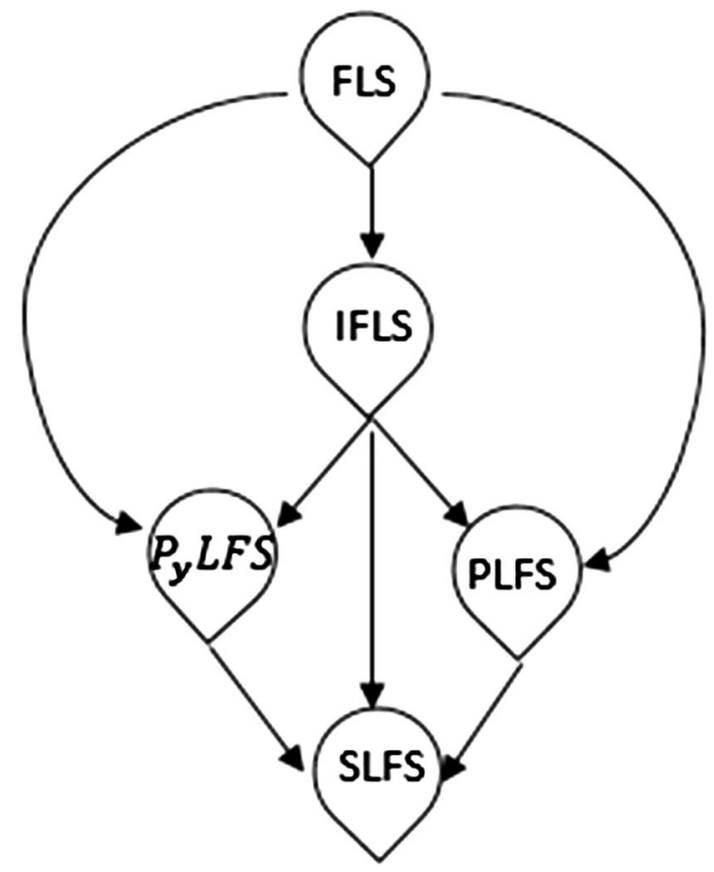

Fig. 2 Hierarchy structure of spherical linguistic fuzzt set

(1) $\breve{E}_{\breve{u}_{1}} \oplus \breve{E}_{\breve{u}_{2}}=\left\{\zeta_{\check{e}_{\check{u}_{1}}+\check{e}_{\breve{u}_{2}}}, \sqrt{P_{\check{e}_{\check{u}_{1}}}^{2}+P_{\check{e}_{\breve{u}_{2}}}^{2}-P_{\check{e}_{\breve{u}_{1}}}^{2} \cdot P_{\check{e}_{\breve{u}_{2}}}^{2}}\right.$, $\left.I_{\check{e}_{\check{u}_{1}}} \cdot I_{\check{e}_{\check{u}_{2}}}, N_{\check{e}_{\check{\iota}_{1}}} \cdot N_{\check{e}_{\tilde{u}_{2}}}\right\}$.

(2) $\tau \cdot \breve{E}_{\breve{u}}=\left\{\zeta_{\tau \cdot \breve{e}_{\breve{u}}}, \sqrt{1-\left(1-P_{\check{e}_{\breve{u}_{1}}}^{2}\right)^{\tau}},\left(I_{\check{e}_{\breve{u}}}\right)^{\tau},\left(N_{\check{e}_{\breve{u}}}\right)^{\tau}\right\}$.

\section{Comparison rules for SLFNs}

Definition 11 Let $\breve{E}_{\breve{u}}=\left\langle\zeta_{\check{\check{u}}_{\breve{u}}}, P_{\breve{e}_{\breve{u}}}, I_{\breve{e}_{\breve{u}}}, N_{\check{e}_{\breve{u}}}\right\rangle$ be any SLFNs. Then,

(1) $\operatorname{sco}\left(\breve{E}_{\breve{u}}\right)=\frac{\zeta_{\check{e}_{\breve{u}}} \times\left(P_{\check{e}_{\breve{u}}}-I_{\check{e}_{\breve{u}}}-N_{\check{e}_{\breve{u}}}\right)}{3}$ which denoted as score function.

(2) $\operatorname{acu}\left(\breve{E}_{\breve{u}}\right)=\frac{\zeta_{\breve{u}}}{2}\left(P_{\check{e}_{\breve{u}}}+N_{\check{e}_{\breve{u}}}\right)$ which is the accuracy function.

(3) $\operatorname{cr}\left(\breve{E}_{\breve{u}}\right)=\frac{\zeta_{\breve{u}}}{2}\left(P_{\breve{e}_{\breve{u}}}\right)$ which is the certainty function.

Idea taken from Definition 11 is the technique used for equating the SLFNs and can be described as

Definition 12 Let $\breve{E}_{\breve{u}_{1}}=\left\langle\zeta_{\check{e}_{\check{u}_{1}}}, P_{\check{e}_{\breve{u}_{1}}}, I_{\check{e}_{\breve{u}_{1}}}, N_{\check{e}_{\check{u}_{1}}}\right\rangle$ and $\breve{E}_{\breve{u}_{2}}=\left\langle\zeta_{\check{e}_{\tilde{u}_{2}}}, P_{\check{e}_{\check{u}_{2}}}, I_{\check{e}_{\breve{u}_{2}}}, N_{\check{e}_{\check{u}_{2}}}\right\rangle$ are two SLFNs defined on the universe of discourse $\mathbb{R} \neq \phi$. Then, by using Definition 11, equating technique can be described as,

(1) If $\operatorname{sco}\left(\breve{E}_{\breve{u}_{1}}\right)>\operatorname{sco}\left(\breve{E}_{\breve{u}_{2}}\right)$, then $\breve{E}_{\breve{u}_{1}}>\breve{E}_{\breve{u}_{2}}$. 
(2) If $\operatorname{sco}\left(\breve{E}_{\breve{u}_{1}}\right) \approx \operatorname{sco}\left(\breve{E}_{\breve{u}_{2}}\right)$, and $\operatorname{acu}\left(\breve{E}_{\breve{u}_{1}}\right)>\operatorname{acu}\left(\breve{E}_{\breve{u}_{2}}\right)$, then $\breve{E}_{\breve{u}_{1}}>\breve{E}_{\breve{u}_{2}}$.

(3) If $\operatorname{sco}\left(\breve{E}_{\breve{u}_{1}}\right) \approx \operatorname{sco}\left(\breve{E}_{\breve{u}_{2}}\right), \quad \operatorname{acu}\left(\breve{E}_{\breve{u}_{1}}\right) \approx \operatorname{acu}\left(\breve{E}_{\breve{u}_{2}}\right) \quad$ and $\operatorname{cr}\left(\breve{E}_{\breve{u}_{1}}\right)>\operatorname{cr}\left(\breve{E}_{\breve{u}_{2}}\right)$, then $\breve{E}_{\breve{u}_{1}}>\breve{E}_{\breve{u}_{2}}$.

(4) If $\operatorname{sco}\left(\breve{E}_{\breve{u}_{1}}\right) \approx \operatorname{sco}\left(\breve{E}_{\breve{u}_{2}}\right), \quad \operatorname{acu}\left(\breve{E}_{\breve{u}_{1}}\right) \approx \operatorname{acu}\left(\breve{E}_{\breve{u}_{2}}\right) \quad$ and $\operatorname{cr}\left(\breve{E}_{\breve{u}_{1}}\right) \approx \operatorname{cr}\left(\breve{E}_{\breve{u}_{2}}\right)$, then $\breve{E}_{\breve{u}_{1}} \approx \breve{E}_{\breve{u}_{2}}$.

Definition 13 Let any collections $\breve{E}_{\breve{u}_{p}}=\left\langle\zeta_{\check{e}_{\check{u}_{p}}}, P_{\check{e}_{\check{u}_{p}}}, I_{\check{e}_{\check{u}_{p}}}, N_{\check{e}_{\check{u}_{p}}}\right\rangle, p \in N$ be the SLFNs and

SLFWA: SLFN ${ }^{n} \rightarrow$ SLFN, then SLFWA is described as,

$\operatorname{SLFWA}\left(\breve{E}_{\breve{u}_{1}}, \breve{E}_{\breve{u}_{2}}, \ldots, \breve{E}_{\breve{u}_{n}}\right)=\sum_{p=1}^{n} \tau_{p} \breve{E}_{\breve{u}_{p}}$

in which $\tau=\left\{\tau_{1}, \tau_{2}, \ldots, \tau_{n}\right\}$ is the weighting vector of $\breve{E}_{\breve{u}_{p}}=\left\langle\zeta_{\check{e}_{\tilde{u}_{p}}}, P_{\check{e}_{\check{u}_{p}}}, I_{\check{e}_{\check{u}_{p}}}, N_{\check{e}_{\check{e}_{p}}}\right\rangle, \quad p \in N$, with $\tau_{p} \geq 0$ and $\sum_{p=1}^{n} \tau_{p}=1$.

Theorem 1 Let any collections $\breve{E}_{\breve{u}_{p}}=\left\langle\zeta_{\check{\check{u}}_{\breve{u}_{p}}}, P_{\check{\check{u}}_{\check{u}_{p}}}, I_{\check{e}_{\check{u}_{p}}}, N_{\check{e}_{\check{u}_{p}}}\right\rangle$, $p \in N$ be the SLFNs. Then, by utilizing Definition 13 and operational properties of SLFNs, we can obtain the following outcome.

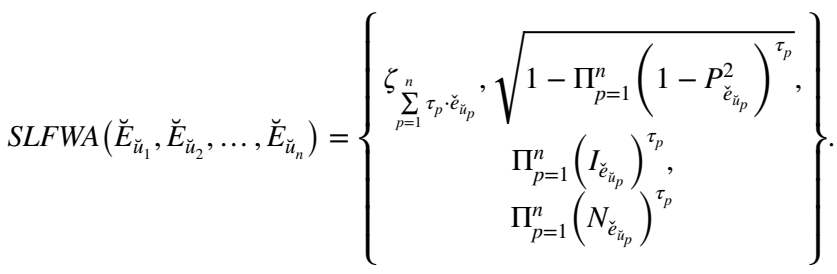

Definition 14 Let any collections $\breve{E}_{\breve{u}_{p}}=\left\langle\zeta_{\check{e}_{\breve{u}_{p}}}, P_{\check{\iota}_{\breve{u}_{p}}}, I_{\check{e}_{\breve{u}_{p}}}, N_{\check{\iota}_{\breve{u}_{p}}}\right\rangle, p \in N$ be the SLFNs and SLFOWA: SLFN ${ }^{n} \rightarrow$ SLFN, then SLFOWA is described as,

$\operatorname{SLFOWA}\left(\breve{E}_{\breve{u}_{1}}, \breve{E}_{\breve{u}_{2}}, \ldots, \breve{E}_{\breve{u}_{n}}\right)=\sum_{p=1}^{n} \tau_{p} \breve{E}_{\breve{u}_{\rho(p)}}$,

in which $\tau=\left\{\tau_{1}, \tau_{2}, \ldots, \tau_{n}\right\}$ is the weighting vector of $\breve{E}_{\breve{u}_{p}}=\left\langle\zeta_{\check{e}_{\check{u}_{p}}}, P_{\check{e}_{\check{u}_{p}}}, I_{\check{\iota}_{\check{u}_{p}}}, N_{\check{e}_{\check{u}_{p}}}\right\rangle, p \in N, \tau_{p} \geq 0$ and $\sum_{p=1}^{n} \tau_{p}=1$, and $\rho(p)$ indicates a permutation on $\mathbb{R}$.

Theorem 2 Let any collections $\breve{E}_{\breve{u}_{p}}=\left\langle\zeta_{\check{e}_{\breve{u}_{p}}}, P_{\check{e}_{\check{u}_{p}}}, I_{\check{e}_{\breve{u}_{p}}}, N_{\check{e}_{\breve{u}_{p}}}\right\rangle$, $p \in N$ be the SLFNs. Then by using Definition 14 and operational properties of SLFNs, we can obtain the following outcome.

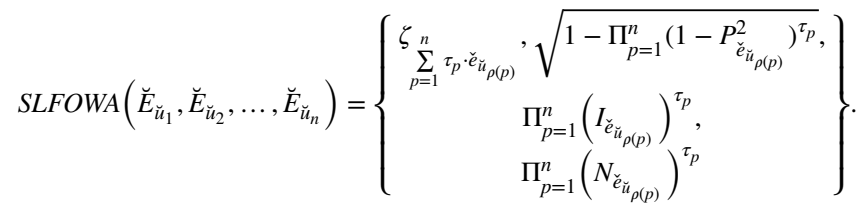

Theorem 3 Assume that $\breve{E}_{\breve{u}_{p}}=\left\langle\zeta_{\check{e}_{\check{u}_{p}}}, P_{\check{e}_{\breve{u}_{p}}}, I_{\check{e}_{\breve{u}_{p}}}, N_{\check{e}_{\check{u}_{p}}}\right\rangle, p \in N$ are the collections of SLFNs and $\lambda$ is a fuzzy measure on $\mathbb{R}$. Based on fuzzy measure, a spherical linguistic fuzzy Choquet integral weighted averaging (SLFCIWA ) operator of dimension $n$ is a mapping SLFCIWA : SLFN ${ }^{n} \rightarrow$ SLFN such that

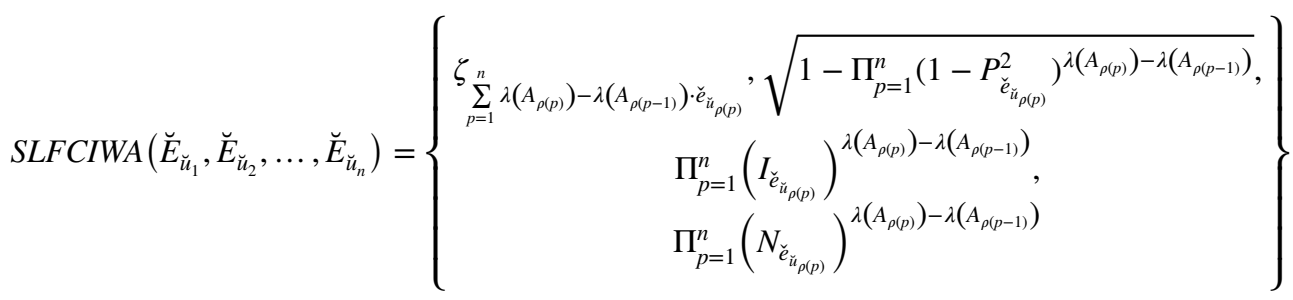


where $\rho(p)$ indicates a permutation on $\mathbb{R}$ and $A_{\rho(n)}=\{1,2, \ldots, p\}, A_{\rho(0)}=\phi$.

Definition 15 Let $\mathbb{R} \neq \phi$ be the universe of discourse and any two spherical linguistic fuzzy sets $\breve{E}_{\breve{u}_{j}}, \breve{E}_{\breve{u}_{l}}$. Then, normalized Hamming distance $d_{\mathrm{NHD}}\left(\breve{E}_{\breve{u}_{j}}, \breve{E}_{\breve{u}_{l}}\right)$ is given as for all $r \in \mathbb{R}$

$d_{\mathrm{NHD}}\left(\breve{E}_{\breve{u}_{j}}, \breve{E}_{\breve{u}_{l}}\right)=\frac{1}{2(l-1)} \sum_{p=1}^{n}\left|\begin{array}{c}\left(P_{\check{e}_{\breve{u}_{j}}}\left(r_{p}\right)-I_{\check{e}_{\breve{u}_{j}}}\left(r_{p}\right)-N_{\check{e}_{\breve{u}_{j}}}\left(r_{p}\right)\right) \zeta_{\check{e}_{\check{u}_{j}}}-\mid \\ \left(P_{\check{e}_{\check{u}_{l}}}\left(r_{p}\right)-I_{\check{e}_{\check{u}_{l}}}\left(r_{p}\right)-N_{\check{e}_{\check{u}_{l}}}\left(r_{p}\right)\right) \zeta_{\check{e}_{\check{u}_{l}}}\end{array}\right|$.

Definition 16 Let $\mathbb{R} \neq \phi$ be the universe of discourse and any two spherical fuzzy sets $\breve{E}_{\breve{u}_{j}}, \breve{E}_{\breve{u}_{l}}$. Then, normalized Euclidean distance $d_{\mathrm{NED}}\left(\breve{E}_{\breve{u}_{j}}, \breve{E}_{\breve{u}_{l}}\right)$ is given as for all $r \in \mathbb{R}$,

$d_{\mathrm{NED}}\left(\breve{E}_{\breve{u}_{j}}, \breve{E}_{\breve{u}_{l}}\right)=\sqrt{\frac{1}{n} \sum_{p=1}^{n}\left(\begin{array}{c}\left.\zeta_{2 \cdot\left(\check{e}_{\breve{u}_{j}}-\check{e}_{\check{u}_{l}}\right)},\left(P_{\check{e}_{\breve{u}_{j}}}\left(r_{p}\right)-P_{\check{e}_{\check{u}_{l}}}\left(r_{p}\right)\right)^{2}+\right) \\ \left(I_{\check{e}_{\breve{u}_{j}}}\left(r_{p}\right)-I_{\check{e}_{\breve{u}_{l}}}\left(r_{p}\right)\right)^{2}+ \\ \left(N_{\check{e}_{\breve{u}_{j}}}\left(r_{p}\right)-N_{\check{e}_{\breve{u}_{l}}}\left(r_{p}\right)\right)^{2}\end{array}\right)}$.

\section{GRA method with incomplete weight information for spherical linguistic fuzzy setting}

Suppose that $A=\left\{a_{1}, a_{2}, \ldots, a_{n}\right\}$ are the set of alternatives and $C=\left\{c_{1}, c_{2}, \ldots, c_{m}\right\}$,are the criteria, and $\vartheta=\left(\vartheta_{1}, \vartheta_{2}, \ldots, \vartheta_{m}\right)$ are the criteria of weight, where $\vartheta_{k} \geq 0$ $(k=1,2, \ldots, m), \Sigma_{k=1}^{n} \vartheta_{k}=1$. Suppose that DM deliver information about weights of criteria may be denoted in the following form [20], for $j \neq k$,

(1) if $\left\{\vartheta_{j} \geq \vartheta_{k}\right\}$, then ranking is weak.

(2) if $\left\{\vartheta_{j}-\vartheta_{k} \geq \lambda_{j}(>0)\right\}$, then ranking is strict.

(3) if $\left\{\vartheta_{j} \geq \lambda_{j} \vartheta_{k}\right\}, 0 \leq \lambda_{j} \leq 1$, then ranking is multiple ranking.

(4) if $\left\{\lambda_{j} \leq \vartheta_{j} \leq \lambda_{j}+\delta_{j}\right\}, 0 \leq \lambda_{j} \leq \lambda_{j}+\delta_{j} \leq 1$, then ranking is an interval ranking.

Let $R^{k}=\left[\breve{E}_{\breve{u}_{q}}^{(k)}\right]_{m \times n}$ be the spherical linguistic fuzzy decision matrix, arranged by decision maker $d_{k}(k=1,2, \ldots, l)$, in the following form:

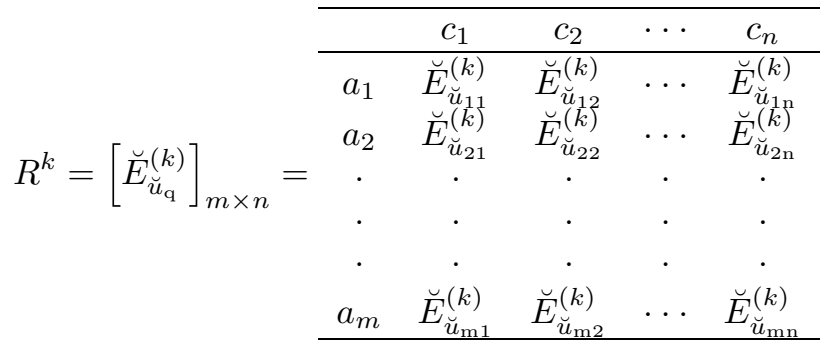

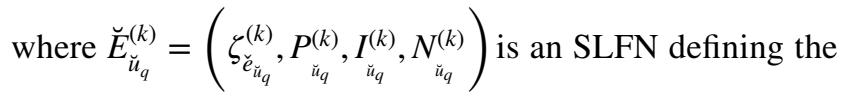
performance rating of the alternative $a_{p} \in A$, with respect to the criteria $c_{p} \in C$ given by the decision makers $d_{k}$. To develop GRA method in the group decision-making process, we first need to combine all individual decision matrices into a collective matrix by using SLFCIWA operator.

Step 1 Suppose that for every $A=\left\{a_{1}, a_{2}, \ldots, a_{m}\right\}, m$ alternative, each expert $d_{k}(k=1,2, \ldots, r)$ is called to consider individual evaluation or preference according to each attributes $C_{q}(q=1,2, \ldots, n)$ by an spherical linguistic fuzzy

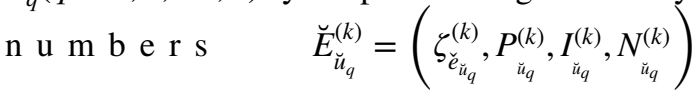
$(p=1,2, \ldots, m ; q=1,2, \ldots, n, k=1,2, \ldots, r)$

expressed by the experts $d_{k}$. In this step, we construct the spherical linguistic fuzzy decision-making matrices, $D^{s}=\left[E_{i p}^{(s)}\right]_{m \times n}(s=1,2, \ldots, k)$ for decision. If we have two types of criteria, such as benefit and cost criteria, in this case spherical linguistic fuzzy decision matrices $D^{s}=\left[E_{i p}^{s}\right]_{m \times n}$ are converted into the normalized spherical linguistic fuzzy decision matrices, $R^{k}=\left[\breve{E}_{\breve{u}_{q}}^{(k)}\right]_{m \times n}$, where

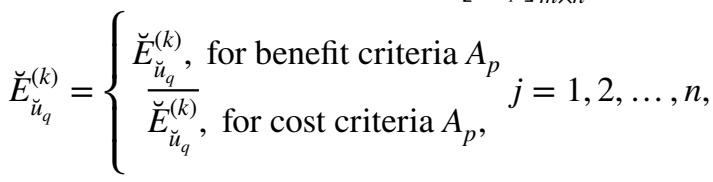
and $\overline{\breve{E}_{\breve{u}_{q}}^{(k)}}$ is the complement of $\breve{E}_{\breve{u}_{q}}^{(k)}$. The use of normalization occur in those cases, the criteria have the alternative type; otherwise, the normalization is not required, and we can write the decisionmaking matrix as follows: 


$$
\begin{aligned}
& \begin{array}{ccccc}
\hline & c_{1} & c_{2} & \cdots & c_{n} \\
\hline a_{1} & \breve{E}_{\breve{u}_{11}}^{(k)} & \breve{E}_{\breve{u}_{12}}^{(k)} & \cdots & \breve{E}_{\breve{u}_{1 \mathrm{n}}}^{(k)}
\end{array}
\end{aligned}
$$

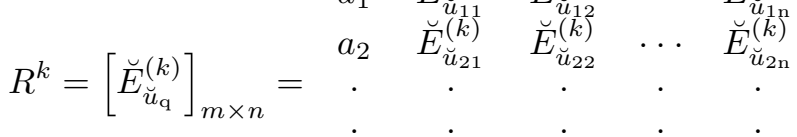

$$
\begin{aligned}
& \begin{array}{lllll}
a_{m} & \breve{E}_{\breve{u}_{\mathrm{m} 1}}^{(k)} & \breve{E}_{\breve{u}_{\mathrm{m} 2}}^{(k)} & \cdots & \breve{E}_{\breve{u}_{\mathrm{mn}}}^{(k)} \\
\hline
\end{array}
\end{aligned}
$$

Step 2 Confirm the fuzzy density $P_{\check{e}_{\breve{u}_{p}}}=P_{\check{e}_{\breve{u}}}\left(a_{p}\right)$ of each professional. According to Eq. (2.11), parameter $\lambda_{1}$ of professional can be calculated.

Step 3 By Definition 7, $\breve{E}_{\breve{u}_{q}}^{(k)}$ is reordered such that $\breve{E}_{\breve{u}_{q}}^{(k)} \geq \breve{E}_{\breve{u}_{q}}^{(k-1)}$. Utilize the spherical linguistic fuzzy

Choquet integral average operator;
Step 5 According to spherical linguistic fuzzy distance operator, find the distance between the alternative $a_{p}$ and the $P^{+}$and $P^{-}$, respectively;

$$
\begin{aligned}
d\left(\breve{E}_{\breve{u}_{j}}, \breve{E}_{\breve{u}_{l}}\right)= & \frac{1}{2(l-1)} \sum_{p=1}^{n} \mid\left(P_{\check{e}_{\breve{u}_{j}}}\left(r_{p}\right)-I_{\check{e}_{\breve{u}_{j}}}\left(r_{p}\right)-N_{\check{e}_{\breve{u}_{j}}}\left(r_{p}\right)\right) \zeta_{\check{e}_{\breve{u}_{j}}} \\
& \quad-\left(P_{\breve{e}_{\breve{u}_{l}}}\left(r_{p}\right)-I_{\breve{e}_{\breve{u}_{l}}}\left(r_{p}\right)-N_{\check{e}_{\breve{u}_{l}}}\left(r_{p}\right)\right) \zeta_{\check{e}_{\breve{u}_{l}}} \mid
\end{aligned}
$$

The name of this distance is normalized Hamming distance [13] $d\left(\breve{E}_{\breve{u}_{j}}, \breve{E}_{\breve{u}_{l}}\right)$,and then construct $D^{+}$(spherical linguistic fuzzy positive-ideal separation matrix) and $D^{-}$(spherical fuzzy negative-ideal separation matrix);

$\operatorname{SLFCIWA}\left(\breve{E}_{\breve{u}_{q}}^{(1)}, \breve{E}_{\breve{u}_{\mathrm{q}}}^{(2)}, \ldots, \breve{E}_{\breve{u}_{q}}^{(r)}\right)=\left\{\begin{array}{c}\zeta_{\sum_{p=1}^{r} \lambda\left(A_{\rho(p)}\right)-\lambda\left(A_{\rho(p-1)}\right) \check{e}_{\breve{u}_{\rho(p)}}}(4.1) \sqrt{1-\Pi_{p=1}^{r}\left(1-P_{\breve{e}_{\breve{u}_{\rho(p)}}}^{2}\right)^{\lambda\left(A_{\rho(p)}\right)-\lambda\left(A_{\rho(p-1)}\right)},} \\ \Pi_{p=1}^{r}\left(I_{\breve{e}_{\breve{u}_{\rho(p)}}}\right)^{\lambda\left(A_{\rho(p)}\right)-\lambda\left(A_{\rho(p-1)}\right)}, \\ \Pi_{p=1}^{r}\left(N_{\breve{e}_{\breve{u}_{\rho(p)}}}\right)^{\lambda\left(A_{\rho(p)}\right)-\lambda\left(A_{\rho(p-1)}\right)}\end{array}\right\}$

to aggregate all the spherical linguistic fuzzy decision matrices $R^{k}=\left[\breve{E}_{\breve{u}_{q}}^{(k)}\right]_{m \times n}(k=1,2, \ldots, r)$ into a collective spherical linguistic fuzzy decision matrix $R=\left[\breve{E}_{\breve{u}_{q}}^{(k)}\right]_{m \times n}$ where $\breve{E}_{\breve{u}_{q}}^{(k)}=\left(\zeta_{\breve{e}_{\breve{u}_{q}}}^{(k)}, P_{\breve{u}_{q}}^{(k)}, I_{\breve{u}_{q}}^{(k)}, N_{\breve{u}_{q}}^{(k)}\right)(p=1,2, \ldots, m ; q=1,2$, $\ldots, n, k=1,2, \ldots, r)$, where $\rho(p)$ indicates a permutation on $\mathbb{R}$ and $A_{\rho(n)}=\{1,2, \ldots, p\}, A_{\rho(0)}=\phi$ and $P_{\breve{e}_{\breve{u}}}\left(a_{p}\right)$ can be determined by Eq. (2.12).

Step 4 The spherical linguistic fuzzy positive-ideal solution $P^{+}=\left\{P_{1}^{+}, P_{2}^{+}, \ldots, P_{m}^{+}\right\}$and the spherical linguistic fuzzy negative-ideal solution $P^{-}=\left\{P_{1}^{-}, P_{2}^{-}, \ldots, P_{m}^{-}\right\}$are defined as

$P_{p}^{+}=\max _{q} s c_{q}$

and

$$
P_{p}^{-}=\min _{q} s c_{q},
$$

$$
\text { w h e r e } \quad P^{+}=\left(\zeta_{\breve{u}_{p}}^{+}, P_{\breve{u}_{p}}^{+}, I_{\breve{u}_{p}}^{+}, N_{\check{u}_{p}}^{+}\right) \quad \text { a n d }
$$

$P^{-}=\left(\zeta_{\breve{u}_{p}}^{-}, P_{\breve{u}_{p}}^{-}, I_{\breve{u}_{p}}^{-}, N_{\breve{u}_{p}}^{-}\right) p=1,2, . ., m$.

$$
D^{+}=\left(D_{q}^{+}\right)_{m \times n}=\left[\begin{array}{cccc}
d\left(\breve{E}_{\breve{u}_{11}}, P_{1}^{+}\right) & d\left(\breve{E}_{\breve{u}_{12}}, P_{2}^{+}\right) & \ldots & d\left(\breve{E}_{\breve{u}_{1 n}}, P_{n}^{+}\right) \\
d\left(\breve{E}_{\breve{u}_{21}}, P_{1}^{+}\right) & d\left(\breve{E}_{\breve{u}_{22}}, P_{2}^{+}\right) & \ldots & d\left(\breve{E}_{\breve{u}_{1 n}}, P_{n}^{+}\right) \\
\cdot & \cdot & & \cdot \\
\cdot & \cdot & \ldots & \cdot \\
\cdot & \cdot & & \cdot \\
d\left(\breve{E}_{\breve{u}_{m 1}}, P_{1}^{+}\right) & d\left(\breve{E}_{\breve{u}_{m 2}}, P_{2}^{+}\right) & \ldots & d\left(\breve{E}_{\breve{u}_{m n}}, P_{n}^{+}\right)
\end{array}\right]
$$

and

$$
D^{-}=\left(D_{q}^{-}\right)_{m \times n}=\left[\begin{array}{cccc}
d\left(\breve{E}_{\breve{u}_{11}}, P_{1}^{-}\right) & d\left(\breve{E}_{\breve{u}_{12}}, P_{2}^{-}\right) & \ldots & d\left(\breve{E}_{\breve{u}_{1 n}}, P_{n}^{-}\right) \\
d\left(\breve{E}_{\breve{u}_{21}}, P_{1}^{-}\right) & d\left(\breve{E}_{\breve{u}_{22}}, P_{2}^{-}\right) & \ldots & d\left(\breve{E}_{\breve{u}_{1 n}}, P_{n}^{-}\right) \\
\cdot & \cdot & & \cdot \\
\cdot & \cdot & \ldots & \cdot \\
\cdot \cdot & \cdot & & \cdot \\
d\left(\breve{E}_{\breve{u}_{m 1}}, P_{1}^{-}\right) & d\left(\breve{E}_{\breve{u}_{m 2}}, P_{2}^{-}\right) & \ldots & d\left(\breve{E}_{\breve{u}_{m n}}, P_{n}^{-}\right)
\end{array}\right]
$$

Step 6 Grey coefficient is calculated for each alternative from PIS and NIS by utilizing the following equation. The grey coefficient for each alternative calculated from PIS is provided as 
Table 1 Spherical linguistic fuzzy information $D^{1}$

\begin{tabular}{lcc}
\hline$\breve{A}_{1}$ & $\breve{A}_{2}$ & $\breve{A}_{3}$ \\
\hline$\check{Z}_{1}\left\langle\zeta_{5}, 0.3,0.8,0.5\right\rangle\left\langle\zeta_{4}, 0.8,0.4,0.3\right\rangle\left\langle\zeta_{2}, 0.4,0.5,0.7\right\rangle\left\langle\zeta_{3}, 0.3,0.3,0.4\right\rangle$ \\
$\check{Z}_{2}\left\langle\zeta_{2}, 0.2,0.6,0.7\right\rangle\left\langle\zeta_{3}, 0.3,0.9,0.1\right\rangle\left\langle\zeta_{1}, 0.5,0.3,0.7\right\rangle\left\langle\zeta_{5}, 0.5,0.4,0.2\right\rangle$ \\
$\check{Z}_{3}\left\langle\zeta_{4}, 0.4,0.8,0.4\right\rangle\left\langle\zeta_{2}, 0.5,0.8,0.2\right\rangle\left\langle\zeta_{5}, 0.2,0.3,0.7\right\rangle\left\langle\zeta_{1}, 0.6,0.6,0.1\right\rangle$ \\
$\check{Z}_{4}\left\langle\zeta_{1}, 0.5,0.3,0.8\right\rangle\left\langle\zeta_{5}, 0.6,0.6,0.3\right\rangle\left\langle\zeta_{3}, 0.3,0.6,0.5\right\rangle\left\langle\zeta_{2}, 0.4,0.2,0.3\right\rangle$ \\
\hline
\end{tabular}

Table 2 Spherical linguistic fuzzy information $D^{2}$

\begin{tabular}{lcc}
\hline$\breve{A}_{1}$ & $\breve{A}_{2}$ & $\breve{A}_{3}$ \\
\hline$\check{Z}_{1}\left\langle\zeta_{2}, 0.1,0.5,0.7\right\rangle\left\langle\zeta_{5}, 0.6,0.3,0.4\right\rangle\left\langle\zeta_{3}, 0.3,0.8,0.6\right\rangle\left\langle\zeta_{4}, 0.6,0.3,0.2\right\rangle$ \\
$\check{Z}_{2}\left\langle\zeta_{5}, 0.4,0.4,0.8\right\rangle\left\langle\zeta_{3}, 0.5,0.7,0.1\right\rangle\left\langle\zeta_{4}, 0.4,0.2,0.7\right\rangle\left\langle\zeta_{2}, 0.6,0.1,0.6\right\rangle$ \\
$\check{Z}_{3}\left\langle\zeta_{3}, 0.2,0.9,0.3\right\rangle\left\langle\zeta_{4}, 0.7,0.1,0.4\right\rangle\left\langle\zeta_{2}, 0.3,0.6,0.4\right\rangle\left\langle\zeta_{5}, 0.6,0.2,0.4\right\rangle$ \\
$\check{Z}_{4}\left\langle\zeta_{1}, 0.3,0.4,0.8\right\rangle\left\langle\zeta_{2}, 0.4,0.6,0.5\right\rangle\left\langle\zeta_{5}, 0.4,0.1,0.8\right\rangle\left\langle\zeta_{3}, 0.6,0.3,0.3\right\rangle$ \\
\hline
\end{tabular}

Table 3 Spherical linguistic fuzzy information $D^{3}$

\begin{tabular}{lcc}
\hline$\breve{A}_{1}$ & $\breve{A}_{2}$ & $\breve{A}_{3}$ \\
\hline$\check{Z}_{1}\left\langle\zeta_{1}, 0.4,0.2,0.8\right\rangle\left\langle\zeta_{3}, 0.4,0.4,0.3\right\rangle\left\langle\zeta_{5}, 0.5,0.4,0.6\right\rangle\left\langle\zeta_{4}, 0.5,0.1,0.4\right\rangle$ \\
$\check{Z}_{2}\left\langle\zeta_{4}, 0.2,0.5,0.7\right\rangle\left\langle\zeta_{5}, 0.6,0.5,0.4\right\rangle\left\langle\zeta_{1}, 0.2,0.5,0.8\right\rangle\left\langle\zeta_{3}, 0.7,0.2,0.4\right\rangle$ \\
$\check{Z}_{3}\left\langle\zeta_{5}, 0.6,0.4,0.5\right\rangle\left\langle\zeta_{2}, 0.9,0.3,0.1\right\rangle\left\langle\zeta_{4}, 0.3,0.1,0.9\right\rangle\left\langle\zeta_{1}, 0.6,0.2,0.6\right\rangle$ \\
$\check{Z}_{4}\left\langle\zeta_{3}, 0.5,0.3,0.7\right\rangle\left\langle\zeta_{4}, 0.8,0.5,0.2\right\rangle\left\langle\zeta_{2}, 0.3,0.8,0.4\right\rangle\left\langle\zeta_{2}, 0.5,0.3,0.5\right\rangle$ \\
\hline
\end{tabular}

Table 4 Normalized spherical linguistic fuzzy information $R^{1}$

\begin{tabular}{lcc}
\hline$\breve{A}_{1}$ & $\breve{A}_{2}$ & $\breve{A}_{3}$ \\
\hline$\check{Z}_{1}\left\langle\zeta_{5}, 0.5,0.8,0.3\right\rangle\left\langle\zeta_{4}, 0.8,0.4,0.3\right\rangle\left\langle\zeta_{2}, 0.7,0.5,0.4\right\rangle\left\langle\zeta_{3}, 0.3,0.3,0.4\right\rangle$ \\
$\check{Z}_{2}\left\langle\zeta_{2}, 0.7,0.6,0.2\right\rangle\left\langle\zeta_{3}, 0.3,0.9,0.1\right\rangle\left\langle\zeta_{1}, 0.7,0.3,0.5\right\rangle\left\langle\zeta_{5}, 0.5,0.4,0.2\right\rangle$ \\
$\check{Z}_{3}\left\langle\zeta_{4}, 0.4,0.8,0.4\right\rangle\left\langle\zeta_{2}, 0.5,0.8,0.2\right\rangle\left\langle\zeta_{5}, 0.7,0.3,0.2\right\rangle\left\langle\zeta_{1}, 0.6,0.6,0.1\right\rangle$ \\
$\check{Z}_{4}\left\langle\zeta_{1}, 0.8,0.3,0.5\right\rangle\left\langle\zeta_{5}, 0.6,0.6,0.3\right\rangle\left\langle\zeta_{3}, 0.5,0.6,0.3\right\rangle\left\langle\zeta_{2}, 0.4,0.2,0.3\right\rangle$ \\
\hline
\end{tabular}

$\eta_{q}^{+}=\frac{\min _{1 \leq p \leq m} \min _{1 \leq q \leq n} d\left(\breve{E}_{\breve{u}_{q}}, P_{p}^{+}\right)+\rho \max _{1 \leq p \leq m} \max _{1 \leq q \leq n} d\left(\breve{E}_{\breve{u}_{q}}, P_{p}^{+}\right)}{d\left(\breve{E}_{\breve{u}_{q}}, P_{p}^{+}\right)+\rho \max _{1 \leq p \leq m} \max _{1 \leq q \leq n} d\left(\breve{E}_{\breve{u}_{q}}, P_{p}^{+}\right)}$.

where $p=1,2,3, \ldots, m$ and $q=1,2,3, \ldots, n$. Similarly, the grey coefficient for each alternative from NIS is provided as

$\eta_{q}^{-}=\frac{\min _{1 \leq p \leq m} \min _{1 \leq q \leq n} d\left(\breve{E}_{\breve{u}_{q}}, P_{k}^{-}\right)+\rho \max _{1 \leq p \leq m} \max _{1 \leq q \leq n} d\left(\breve{E}_{\breve{u}_{q}}, P_{k}^{-}\right)}{d\left(\breve{E}_{\breve{u}_{q}}, P_{k}^{-}\right)+\rho \max _{1 \leq p \leq m} \max _{1 \leq q \leq n} d\left(\breve{E}_{\breve{u}_{q}}, P_{k}^{-}\right)}$.

where $p=1,2,3, \ldots, m$ and $q=1,2,3, \ldots, n$ and the identification coefficient $\rho=0.5$.

Step 7 Use Eq. (2.7), to find the grey coefficient degree for each alternative from PIS and NIS, respectively,

$$
\begin{aligned}
& \eta_{p}^{+}=\sum_{q=1}^{n} \vartheta_{q} \eta_{q}^{+} \\
& \eta_{p}^{-}=\sum_{q=1}^{n} \vartheta_{q} \eta_{q}^{-}
\end{aligned}
$$

The grey method has the following basic principle that the selected alternative should have the "biggest degree of grey relation" from the PIS and the "smallest degree of grey relation" from the NIS. Clearly, the weights are known, the smaller $\eta_{p}^{-}$and the bigger $\eta_{p}^{+}$, the finest alternative $a_{p}$ is. But information about weights of alternatives is unknown. So, in this circumstances the $\eta_{p}^{-}$and $\eta_{p}^{+}$are information about weight calculated initially. So, we provide the following optimization models for multiple objective to calculate the information about weight,

Table 5 Normalized spherical linguistic fuzzy information $R^{2}$

\begin{tabular}{lcc}
\hline$\breve{A}_{1}$ & $\breve{A}_{2}$ & $\breve{A}_{3}$ \\
\hline$\check{Z}_{1}\left\langle\zeta_{2}, 0.7,0.5,0.1\right\rangle\left\langle\zeta_{5}, 0.6,0.3,0.4\right\rangle\left\langle\zeta_{3}, 0.6,0.8,0.3\right\rangle\left\langle\zeta_{4}, 0.6,0.3,0.2\right\rangle$ \\
$\check{Z}_{2}\left\langle\zeta_{5}, 0.8,0.4,0.4\right\rangle\left\langle\zeta_{3}, 0.5,0.7,0.1\right\rangle\left\langle\zeta_{4}, 0.7,0.2,0.4\right\rangle\left\langle\zeta_{2}, 0.6,0.1,0.6\right\rangle$ \\
$\check{Z}_{3}\left\langle\zeta_{3}, 0.3,0.9,0.2\right\rangle\left\langle\zeta_{4}, 0.7,0.1,0.4\right\rangle\left\langle\zeta_{2}, 0.4,0.6,0.3\right\rangle\left\langle\zeta_{5}, 0.6,0.2,0.4\right\rangle$ \\
$\check{Z}_{4}\left\langle\zeta_{1}, 0.8,0.4,0.3\right\rangle\left\langle\zeta_{2}, 0.4,0.6,0.5\right\rangle\left\langle\zeta_{5}, 0.8,0.1,0.4\right\rangle\left\langle\zeta_{3}, 0.6,0.3,0.3\right\rangle$ \\
\hline
\end{tabular}

Table 6 Normalized spherical linguistic fuzzy information $R^{3}$

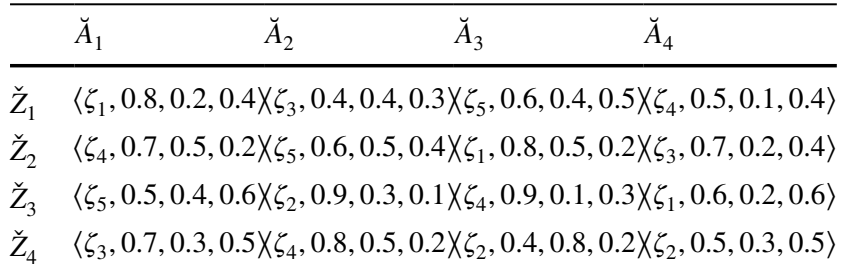


Table 7 Collective spherical linguistic fuzzy information

\begin{tabular}{lllll}
\hline & $\breve{A}_{1}$ & $\breve{A}_{2}$ & $\breve{A}_{3}$ & $\breve{A}_{4}$ \\
\hline$\check{Z}_{1}$ & $\left\langle\zeta_{2.55}, 0.702,0.417,0.225\right\rangle$ & $\left\langle\zeta_{4.00}, 0.638,0.361,0.331\right\rangle$ & $\left\langle\zeta_{3.40}, 0.634,0.545,0.391\right\rangle$ & $\left\langle\zeta_{3.75}, 0.498,0.204,0.313\right\rangle$ \\
$\check{Z}_{2}$ & $\left\langle\zeta_{3.75}, 0.740,0.488,0.254\right\rangle$ & $\left\langle\zeta_{3.70}, 0.498,0.670,0.162\right\rangle$ & $\left\langle\zeta_{2.05}, 0.740,0.311,0.335\right\rangle$ & $\left\langle\zeta_{3.25}, 0.616,0.193,0.374\right\rangle$ \\
$\check{Z}_{3}$ & $\left\langle\zeta_{4.00}, 0.411,0.654,0.361\right\rangle$ & $\left\langle\zeta_{2.70}, 0.748,0.274,0.200\right\rangle$ & $\left\langle\zeta_{3.60}, 0.755,0.260,0.265\right\rangle$ & $\left\langle\zeta_{2.40}, 0.600,0.278,0.304\right\rangle$ \\
$\check{Z}_{4}$ & $\left\langle\zeta_{1.70}, 0.770,0.331,0.418\right\rangle$ & $\left\langle\zeta_{3.60}, 0.651,0.562,0.311\right\rangle$ & $\left\langle\zeta_{3.35}, 0.629,0.354,0.311\right\rangle$ & $\left\langle\zeta_{2.35}, 0.515,0.265,0.358\right\rangle$ \\
\hline
\end{tabular}

$(\mathrm{OM} 1)\left\{\begin{array}{l}\min \eta_{p}^{-}=\sum_{q=1}^{n} \vartheta_{q} \eta_{q}^{-} p=1,2, \ldots, m \\ \max \eta_{p}^{+}=\sum_{q=1}^{n} \vartheta_{q} \eta_{q}^{+} p=1,2, \ldots, m\end{array}\right.$

Since it is given that each alternative is non-inferior, therefore all the alternatives have no preference relation. The above optimization models are aggregated with equal weights, into single-objective optimization model,

$(\mathrm{OM} 2)\left\{\min \eta_{p}=\sum_{p=1}^{m} \sum_{q=1}^{n}\left(\eta_{q}^{-}-\eta_{q}^{+}\right) \vartheta_{q}\right.$

To find solution of OM2, we obtain optimal solution $\vartheta=\left(\vartheta_{1}, \vartheta_{2}, \ldots, \vartheta_{m}\right)$, which is utilized as weight information of provided alternatives. Then, we obtain $\eta_{p}^{+}$and $\eta_{p}^{-}$(where $p=1,2, \ldots, m)$ utilizing the above formula, respectively.

Step 8 Relative degree is calculated for each alternative utilizing the following equation from PIS and NIS,

$\eta_{p}=\frac{\eta_{p}^{+}}{\eta_{p}^{-}+\eta_{p}^{+}}$

Step 9 Rank the alternatives $a_{p}$ and choose finest ones, in according with the $\eta_{p}$, and the highest $\eta_{p}$ alternative is considered as the finest alternative according to the criteria.

Step 10 End.

\section{Descriptive example}

Example 1 Suppose that there are a four possible developed technology business $\check{Z}_{i}(i=1,2,3,4)$ forum. The number of experts is three, and the number attributes is four, to calculate the four desirable emerging technology business:

(1) $\breve{A}_{1}$ is the industrial growth;

(2) $\breve{A}_{2}$ is the possible market risk;

(3) $\breve{A}_{3}$ is the industrialization infrastructure, human resources and financial situation;

(4) $\breve{A}_{4}$ is the job production and the improvement of science and technology.
Table 8 Positive-ideal separation matrix

\begin{tabular}{|c|c|c|c|c|c|}
\hline & & $\breve{A}_{1}$ & $\breve{A}_{2}$ & $\breve{A}_{3}$ & $\breve{A}_{4}$ \\
\hline \multirow[t]{4}{*}{$D^{+}=$} & $\check{Z}_{1}$ & 0.0000 & 0.0799 & 0.1543 & 0.0193 \\
\hline & $\check{Z}_{2}$ & 0.0133 & 0.1650 & 0.0527 & 0.0000 \\
\hline & $\check{Z}_{3}$ & 0.2137 & 0.0000 & 0.0000 & 0.0096 \\
\hline & $\check{Z}_{4}$ & 0.0095 & 0.1284 & 0.0788 & 0.0345 \\
\hline
\end{tabular}

Table 9 Negative-ideal separation matrix

\begin{tabular}{|c|c|c|c|c|c|}
\hline & & $\breve{A}_{1}$ & $\breve{A}_{2}$ & $\breve{A}_{3}$ & $\breve{A}_{4}$ \\
\hline \multirow[t]{4}{*}{$D^{-}=$} & $\check{Z}_{1}$ & 0.2137 & 0.0850 & 0.0000 & 0.0151 \\
\hline & $\check{Z}_{2}$ & 0.2004 & 0.0000 & 0.1016 & 0.0345 \\
\hline & $\check{Z}_{3}$ & 0.0000 & 0.1650 & 0.1543 & 0.0248 \\
\hline & $\check{Z}_{4}$ & 0.2041 & 0.0365 & 0.0755 & 0.0000 \\
\hline
\end{tabular}

Step 1 The three experts offering their own opinions with respect to the results gained with each emerging technology enterprise are given in Tables 1,2 and 3 .

$C_{1}, C_{3}$ are cost-type criteria and $C_{2}, C_{4}$ are benefit-type criteria. So, we have need to normalized the spherical linguistic fuzzy information. So normalized spherical linguistic fuzzy information is given in Tables 4, 5 and 6. Let us assume that the information of the attribute weights is given by experts and is partly known; $\Delta=\left\{0.2 \leq w_{1} \leq 0.25,0.15 \leq w_{2} \leq 0.2,0.28 \leq w_{3} \leq 0.32\right.$, $\left.0.35 \leq w_{4} \leq 0.4\right\}, w_{p} \geq 0, \quad p=1,2,3,4, \quad \sum_{p=1}^{4} w_{p}=1$ Then, we utilize the developed approach to draw the most beautiful alternative(s).

Step 2 Find for each decision maker their fuzzy density, where $\lambda$ is its parameter. Assume that $P_{\breve{e}_{\breve{u}}}\left(A_{1}\right)=0.30$, $P_{\breve{e}_{\breve{u}}}\left(A_{2}\right)=0.40, P_{\breve{e}_{\breve{u}}}\left(A_{3}\right)=0.50$. Then, $\lambda$ of expert can be determined, which is $\lambda=-0.45$. By Eq. (2.9), we have $P_{\check{e}_{\breve{u}}}\left(A_{1}, A_{2}\right)=0.65, P_{\breve{e}_{\breve{u}}}\left(A_{1}, A_{3}\right)=0.73, P_{\breve{e}_{\breve{u}}}\left(A_{2}, A_{3}\right)=0.81$, $P_{\check{e}_{\breve{u}}}\left(A_{1}, A_{2}, A_{3}\right)=1$.

Step 3 According to Definition 12, $\breve{E}_{\breve{u}_{q}}^{(k)}$ is reordered such that $\breve{E}_{\breve{u}_{q}}^{(k)} \geq \breve{E}_{\breve{u}_{q}}^{(k-1)}$. Then, utilize the spherical fuzzy linguistic Choquet integral weighted averaging operator 
$\operatorname{SLFCIWA}\left(\breve{E}_{\breve{u}_{1}}, \breve{E}_{\breve{u}_{2}}, \ldots, \breve{E}_{\breve{u}_{n}}\right)=\left\{\begin{array}{c}\zeta_{p=1}^{r} \lambda\left(A_{\rho(p)}\right)-\lambda\left(A_{\rho(p-1)}\right) \cdot \check{\check{e}}_{\breve{\rho}_{\rho(p)}}, \sqrt{1-\Pi_{p=1}^{n}\left(1-P_{\check{e}_{\breve{p}_{\rho(p)}}^{2}}^{2}\right)^{\lambda\left(A_{\rho(p)}\right)-\lambda\left(A_{\rho(p-1)}\right)}}, \\ \prod_{p=1}^{n}\left(I_{\check{e}_{\breve{u}_{\rho(p)}}}\right)^{\lambda\left(A_{\rho(p)}\right)-\lambda\left(A_{\rho(p-1)}\right)}, \Pi_{p=1}^{n}\left(N_{\check{e}_{\breve{u}_{\rho}(p)}}\right)^{\lambda\left(A_{\rho(p)}\right)-\lambda\left(A_{\rho(p-1)}\right)}\end{array}\right\}$

to aggregate all the spherical linguistic fuzzy decision matrices $R^{k}=\left[\breve{E}_{\breve{u}_{q}}^{(k)}\right]_{m \times n}$ into a cumulative spherical linguistic fuzzy decision matrix as follows (Table 7):

Step 4 Utilizing Eqs. (4.2) and (4.3), we get the positiveideal and negative-ideal solutions, respectively,

$$
\begin{aligned}
& P^{+}=\left\{\begin{array}{l}
\left\langle\zeta_{2.55}, 0.702,0.417,0.225\right\rangle,\left\langle\zeta_{2.70}, 0.748,0.274,0.200\right\rangle, \\
\left\langle\zeta_{3.60}, 0.755,0.260,0.265\right\rangle,\left\langle\zeta_{3.25}, 0.616,0.193,0.374\right\rangle
\end{array}\right\} \\
& P^{-}=\left\{\begin{array}{l}
\left\langle\zeta_{4.00}, 0.411,0.654,0.361\right\rangle,\left\langle\zeta_{3.70}, 0.498,0.670,0.162\right\rangle, \\
\left\langle\zeta_{3.40}, 0.634,0.545,0.391\right\rangle,\left\langle\zeta_{2.35}, 0.515,0.265,0.358\right\rangle
\end{array}\right\}
\end{aligned}
$$

Step 5 Utilize Eqs. (4.5) and (4.6) to get the positiveideal separation matrix $\left(D^{+}\right)$and negative-ideal separation matrix $\left(D^{-}\right)$, respectively (Tables 8,9 );

Step 6 Utilize Eqs. (4.7) and (4.8) to get the grey relational coefficient matrices in which each alternative is calculated from PIS and NIS as follows:

$$
\begin{aligned}
{\left[\eta_{i j}^{+}\right.} & =\left[\begin{array}{llll}
1.0000 & 0.5721 & 0.4091 & 0.8470 \\
0.8893 & 0.3930 & 0.6696 & 1.0000 \\
0.3333 & 1.0000 & 1.0000 & 0.9175 \\
0.9183 & 0.4541 & 0.5755 & 0.7559
\end{array}\right] \\
{\left[\eta_{i j}^{-}\right.} & =\left[\begin{array}{llll}
0.3333 & 0.5569 & 1.0000 & 0.8761 \\
0.3477 & 1.0000 & 0.5125 & 0.7559 \\
1.0000 & 0.3930 & 0.4091 & 0.8116 \\
0.3436 & 0.7453 & 0.5859 & 1.0000
\end{array}\right]
\end{aligned}
$$

Step 7 Use the model (M2) to form the single-objective programming model, which is:

$\min \eta(w)=-0.0619 w_{1}-0.3358 w_{2}-0.6371 w_{3}-0.0290 w_{4}$ After the solution of the model, the weighting vector of attributes is:

$w=(0.226,0.416,0.312,0.043)$

Then, for each alternative, we obtain the degree of grey relational coefficient from PIS and NIS:

$\eta_{1}^{+}=0.6280, \eta_{2}^{+}=0.6163, \eta_{3}^{+}=0.8427, \eta_{4}^{+}=0.6085$,

$\eta_{1}^{-}=0.6566, \eta_{2}^{-}=0.6869, \eta_{3}^{-}=0.5520, \eta_{4}^{-}=0.6134$.
Step 8 Utilize Eq. 4.12, on the PIS and NIS, to obtain the relative relational degree of each alternative, which are the following:

$$
\begin{aligned}
& \eta_{1}=\frac{\eta_{1}^{+}}{\eta_{1}^{-}+\eta_{1}^{+}}=\frac{0.6280}{0.6566+0.6280}=0.4888 \\
& \eta_{2}=\frac{\eta_{2}^{+}}{\eta_{2}^{-}+\eta_{2}^{+}}=\frac{0.6163}{0.6869+0.6163}=0.4729 \\
& \eta_{3}=\frac{\eta_{3}^{+}}{\eta_{3}^{-}+\eta_{3}^{+}}=\frac{0.8427}{0.5520+0.8427}=0.6042 \\
& \eta_{4}=\frac{\eta_{4}^{+}}{\eta_{4}^{-}+\eta_{4}^{+}}=\frac{0.6085}{0.6134+0.6085}=0.4979
\end{aligned}
$$

Step 9 Rank the alternatives, with the relative relational degree follows:

$\check{Z}_{3}>\check{Z}_{4}>\check{Z}_{1}>\check{Z}_{2}$,

and so the most suitable alternative is $\check{Z}_{3}$.

Step 10 End.

\section{Comparative analysis}

To verify the effectively and efficiency of the suggested technique, we conducted a comparative study for comparison of our suggested technique with the Pythagorean fuzzy TOPSIS method [38].

\section{A comparison analysis with the pythagorean fuzzy TOPSIS}

These two techniques easily tackle the MAGDM problems, grey method (GRA) firstly introduced by Deng [14] to execute a multi-attribute performance scheme as a tool which is utilized to recognize solutions from a finite alternative set. TOPSIS, firstly defined by Hwang and Yoon [18], is the modest and suitable path to resolve the MAGDM problems with using crisp sets, purposes of which is that it elects that alternative which has the smallest distance and the utmost distance from the positive-ideal solution and negative-ideal solution, respectively.

To rank results the alternatives we used the Pythagorean fuzzy GRA method and the Pythagorean fuzzy TOPSIS 
technique are clearly dissimilar. The Pythagorean fuzzy TOPSIS method can be used only in the circumstances where the DMs are completely analytical. After all, in practice, for the incomplete information or some other factors, the DMs cannot generally deliver the precise preferences. In other words, the DMs are not rational to some grade. The Pythagorean fuzzy GRA method can sensibly characterize the DMs' performances under risk, and consequently it may tackle above subject excellently.

\section{Conclusion}

The classic grey relational analysis procedure is usually appropriate for tackling the MAGDM problems, where the data are in the form of numerical values. If MAGDM problems contain spherical linguistic fuzzy information, then classic grey relational analysis procedure is flopped to handle such a situation. In this article, we launch the multiple objective optimization representations on the basis of elementary classical GRA method. In the proposed method, we use the spherical linguistic fuzzy Choquet integral weighted averaging (SLFCIWA) operator to merge all the separate matrices. Then, on the basis of the traditional GRA method, calculation steps for dealing with spherical linguistic fuzzy MAGDM problems with incomplete information are given. Finally, a decision problem has established which is based on these defined operators, to rank the dissimilar alternatives for spherical linguistic fuzzy setting. The implied approach has been exposed with a numerical example to observe its success along with reliableness. Thus, the proposed operations give a new easier track to grip the inexact data throughout the decision problem procedure.

Open Access This article is distributed under the terms of the Creative Commons Attribution 4.0 International License (http://creativeco mmons.org/licenses/by/4.0/), which permits unrestricted use, distribution, and reproduction in any medium, provided you give appropriate credit to the original author(s) and the source, provide a link to the Creative Commons license, and indicate if changes were made.

\section{References}

1. Adamopoulos, G.I., Pappis, G.P.: A fuzzy linguistic approach to a multi-criteria sequencing problems. Eur. J. Oper. Res. 92, 628-636 (1996)

2. Ashraf, S., Mahmood, T., Abdullah, S., Khan, Q.: Different approaches to multi-criteria group decision making problems for picture fuzzy environment. Bull. Braz. Math. Soc. New Ser. (2018). https://doi.org/10.1007/s00574-018-0103-y

3. Ashraf, S., Mahmood, T., Khan, Q.: Picture fuzzy linguistic sets and their applications for multi-attribute group decision making problems. Nucleus 55(2), 66-73 (2018)

4. Ashraf, S., Abdullah, S., Qadir, A.: Novel concept of cubic picture fuzzy sets. J. New Theory 24, 59-72 (2018)
5. Atanassov, K.T.: Operations over interval-valued fuzzy set. Fuzzy Sets Syst. 64, 159-174 (1994)

6. Atanassov, K.T.: Intuitionistic fuzzy logics as tools for evaluation of data mining processes. Knowl-Based Syst. 80, 122-130 (2015)

7. Choquet, G.: Theory of capacities. Ann. Inst. Fourier 5, 131-295 (1954)

8. Cuong, B.C.: Picture fuzzy sets -first results. part 1, seminar neuro-fuzzy systems with applications. Technical Report, Institute of Mathematics, Hanoi (2013)

9. Cuong, B.C.: Picture fuzzy sets -first results. part 2, seminar neuro-fuzzy systems with applications. Technical Report, Institute of Mathematics, Hanoi (2013)

10. Cuong, B.C.: Picture fuzzy sets. J. Comput. Sci. Cybern. 30(4), 409-420 (2014)

11. Cuong, B.C., Phan, V.H.: some fuzzy logic operations for picture fuzzy sets. In: Preceding of Seventh International Conference on Knowledge and System Engineering (IEEE) (2015)

12. Chen, Z., Liu, P., Pei, Z.: An approach to multiple attribute group decision making based on linguistic intuitionistic fuzzy numbers. Int. J. Comput. Intell. Syst. 8(4), 747-760 (2015)

13. Cuong, B.C., Kreinovitch, V., Ngan, R.T.: A classification of representable t-norm operators for picture fuzzy sets. In: Eighth International Conference on Knowledge and Systems Engineering (2016)

14. Deng, J.L.: Introduction to grey system theory. Grey Syst. 1, 1-24 (1989)

15. Garg, H.: Some picture fuzzy aggregation operators and their applications to multicriteria decision-making. Arab. J. Sci. Eng. 42, 5275 (2017)

16. Herrera, F., Herrera-Viedma, E., Martinez, L.: A fusion approach for managing multigranularity linguistic term sets in decision making. Fuzzy Sets Syst. 114(1), 43-58 (2000)

17. Herrera, F., Martinez, L.: A 2-tuple fuzzy linguistic representation model for computing with words. IEEE Trans. Fuzzy Syst. 8(6), 746-752 (2000)

18. Hwang, C.L., Yoon, K.: Multiple Attribute Decision Making: Methods and Applications. Springer, New York (1981) https:// doi.org/10.1007/978-3-642-48318-9

19. Khan, M.S.A., Ali, M.Y., Abdullah, S., Hussain, I., Farooq, M.: Extension of TOPSIS method base on Choquet integral under interval-valued Pythagorean fuzzy environment. J. Intell. Fuzzy Syst. (2018). https://doi.org/10.3233/JIFS-171164

20. Kim, S.H., Ahn, B.S.: Interactive group decision making procedure under incomplete information. Eur. J. Oper. Res. 116, 498-507 (1999)

21. Liu, P.D.: Some generalized dependent aggregation operators with intuitionistic linguistic numbers and their application to group decision making. J. Comput. Syst. Sci. 79(1), 131-143 (2013)

22. Liu, P.D., Wang, Y.M.: Multi attribute group decision making method based on intuitionistic linguistic power generalized aggregation operators. Appl. Soft Comput. 17, 90-104 (2014)

23. Merigó, J.M., Gil-Lafuente, A.M.: Induced 2-tuple linguistic generalized aggregation operators and their application in decisionmaking. Inf. Sci. 236, 1-16 (2013)

24. Ma, Z., Xu, Z.: Symmetric Pythagorean fuzzy weighted geometric/averaging operators and their application to multi-criteria decision making problems. Int. J. Intell. Syst. 31(12), 1198-1219 (2016)

25. Pei, Z., Shi, P.: Fuzzy risk analysis based on linguistic aggregation operators. Int. J. Innov. Comput. Inf. Control 7, 7105-7118 (2011)

26. Peng, X.D., Yang, Y.: Some results for Pythagorean fuzzy sets. Int. J. Intell. Syst. 30, 1133-1160 (2015)

27. Phong, P.H., Cuong, B.C.: Multi-criteria group decision making with picture linguistic numbers. VNU J. Sci. Comput. Sci. Comput. Eng. 32(3), 39-53 (2017) 
28. Singh, P.: Correlation coeficients for picture fuzzy sets. J. Intell. Fuzzy Syst. 28, 591-604 (2015)

29. Son, L.H.: Generalized picture distance measure and applications to picture fuzzy clustering. Appl. Soft Comput. 46, 284-295 (2016)

30. Sugeno, M.: Theory of fuzzy integral and its application (Doctoral dissertation), Tokyo Institute of technology, Tokyo, Japan (1974)

31. Wang, X.F., Wang, J.Q., Yang, W.E.: Multi-criteria group decision making method based on intuitionistic linguistic aggregation operators. J. Intell. Fuzzy Syst. 26(1), 115-125 (2014)

32. Wei, G.W.: GRA method for multiple attribute decision making with incomplete weight information in intuitionistic fuzzy setting. Knowl. Based Syst. 23, 243-247 (2010)

33. Wei, G.W.: Gray relational analysis method for intuitionistic fuzzy multiple attribute decision making. Expert Syst. Appl. 38, 11671-11677 (2011)

34. Wei, G.W.: Picture fuzzy cross-entropy for multiple attribute decision making problems. J. Bus. Econ. Manag. 17(4), 491-502 (2016)
35. Yager, R.R.: Pythagorean fuzzy subsets. In: Proceedings of the Joint IFSA World Congress and NAFIPS Annual Meeting, Edmonton, Canada, 24.28 June, pp. 57-61 (2013)

36. Yager, R.R.: Pythagorean membership grades in multicriteria decision making. IEEE Trans. Fuzzy Syst. 22, 958-965 (2014)

37. Zadeh, L.A.: Fuzzy collection. Inf. Control 8, 338-356 (1965)

38. Zhang, X.L., Xu, Z.S.: Extention of TOPSIS to multiple criteria decision making with Pythagorean fuzzy sets. Int. J. Intell. Syst. 29, 1061-1078 (2014)

39. Zhao, H., Xu, Z., Ni, M.F., Liu, S.: Generalized aggregation operators for intuitionistic fuzzy sets. Int. J. Intell. Syst. 25(1), 1-30 (2010)

Publisher's Note Springer Nature remains neutral with regard to jurisdictional claims in published maps and institutional affiliations. 